\title{
Severe Falciparum Malaria: An Overview
}

\author{
Khalid Abdelsamea Mohamedahmed ${ }^{1,2^{*}}$, Adam Dawoud Abakar ${ }^{3^{\circledR}}$ \\ ${ }^{1}$ Department of Hematology, Faculty of Medical Laboratory Science, University of Gezira, Wad Medani, Sudan \\ ${ }^{2}$ Department of Immunology, Faculty of Medical Laboratory Science, University of Gezira, Wad Medani, Sudan \\ ${ }^{3}$ Department of Medical Parasitology, Faculty of Medical Laboratory Science, University of Gezira, Wad Medani, Sudan
}

$\mathrm{M}$ alaria is a human intracellular protozoan parasitic disease caused by female anopheline mosquitoes inoculating plasmodium parasites on erythrocytes (1). Five types of Plasmodium are known to cause diseases in humans: $P$. falciparum, $P$. vivax, P. malariae, P. ovale, and P. knowlesi (2). Malaria is one of the leading causes of morbidity and death in the world, especially in Africa (3). In 2017, there were 219 million cases of malaria, responsible for 435000 deaths worldwide. Malaria affects children aged 1-5 years the most, accounting for $61 \%$ (266000) of all malaria deaths. In 2017, the WHO African Region had the highest number of malaria cases (200 million or 92\%) and accounted for $93 \%$ of all malaria deaths. India and most countries in Sub-Saharan Africa bore nearly $80 \%$ of the global burden of the disease. P. falciparum is the most common malaria parasite in Africa (4).

Falciparum malaria is the most common malaria (5). In 2017, falciparum malaria accounted for $99.7 \%$ of malaria cases in Africa, 62.8\% in South-East Asia, 69\% in the Eastern Mediterranean Region, and 71.9\% in the Western Pacific Region (4). Falciparum malaria is responsible for the vast majority of deaths (99\%) (6).

Plasmodium falciparum is the most pathogenic type of human malaria parasite, and it is linked to acute parasitemia, which can lead to severe or complicated infection and death in infants, pregnant women, and nonimmune individuals, as well as higher parasite burdens, and dysfunction of vital organs $(7,8)$. If no effective therapy is administered promptly, mortality rates of about $25 \%$ and $30 \%$ will be expected in non-immune adults and non-immune infants, respectively (1).

Even though falciparum malaria is unrestricted to erythrocytes of a specific age, it is associated with the highest degree of parasite density. Falciparum malaria may also cause vascular obstruction which results from its ability to bind to endothelial cell walls. The majority of the complications of $P$. falciparum infection occur due to this phenomenon (9).

The majority of malaria deaths occur due to Falciparum malaria. The infection of red blood cells (RBCs) with the mature plasmodium parasite results in the expression of parasite proteins, which is associated with their morphological structures ("knobs") that permit them to bind to endothelial cells lining the blood vessels and lead to sequestration by vascular bed of vital organs of these infected RBCs. Cerebral malaria causes brain injury, which can lead to coma and death. Complications may involve the kidneys, lungs, and gastrointestinal tract. Stillbirth, low birth weight, and prenatal mortality are all possible outcomes of congenital malaria and placental infection. Repeated infections of erythrocytes by merozoites after the parasite initiates blood stage infection results in exponential development. As a result, the parasitized RBCs accumulate in capillaries and sinusoids, causing obstruction of peripheral blood circulation. Organomegaly, especially in the spleen and liver, is caused by congestion, which leads to anemia, leucopenia, and thrombocytopenia (7).

The severity of falciparum malaria is assessed by the presence of many factors such as the infective dose of the parasite, the host's nutritional status, immune system status, growth rate of the parasite, genetic factors, socioeconomic status, antimalarial drug resistance status, access to health care, and education (10).

Coma, respiratory distress (pulmonary edema), bleeding, shock, intravascular hemolysis, jaundice, convulsions (clinical manifestations), severe anemia, hypoglycemia, Lactic (metabolic) acidosis, renal failure, and hyperparasitemia (laboratory features) are among the criteria recommended by the World Health Organization (WHO) for classifying severe malaria infections. Severe malaria is diagnosed based on the presence of two or more clinical or laboratory findings (12). Cerebral 
malaria, hemoglobinuria, hypotension, hematological changes (such as thrombocytopenia), hepatic instability, and cardiovascular failure are all correlated with severe falciparum malaria (2). Malaria caused by P. falciparum is associated with a mortality rate of 7\%-32\%, depending on the organs and systems involved (12).

Severe falciparum malaria is primarily a childhood disease in areas of the world where transmission of $P$. falciparum is intense and stable, and it is less frequent in adults and older children as unique adaptive immunity provides developed (though still incomplete) protection. Sub-Sahara Africa has the highest number of malaria cases, and $90 \%$ of the world's malaria-related deaths occur in this region (13).

We concluded that the falciparum malaria, especially its severe form, is one of the most common reasons for hospital admission and one of the most common causes of young children death in hospital in several endemic areas especially in sub-Saharan Africa. Finally, we recommended that severe falciparum malaria should be diagnosed using WHO criteria (presence of malaria parasitemia $(+)$ and $(++)$ did not roll out the severe malaria, and hyperparasitemia did not roll out the uncomplicated malaria).

\section{Conflict of Interests}

The authors have declared that no competing interests exist.

\section{Ethical Issues}

Not applicable.

\section{Authors' Contributions}

KAM developed the concepts, performed literature search, and prepared and edited the manuscript. ADA prepared and reviewed the article draft.

\section{Funding}

This study received no specific grant from any funding agency in the public, commercial or non-profit sectors.

\section{References}

1. Recker M, Bull PC, Buckee CO. Recent advances in the molecular epidemiology of clinical malaria. F1000Res. 2018;7. doi:10.12688/f1000research.14991.1

2. World Health Organization (WHO). Guidelines for the Treatment of Malaria. 3rd ed. Geneva: WHO; 2015.

3. Keiser J, Utzinger J, Caldas de Castro M, Smith TA, Tanner $\mathrm{M}$, Singer BH. Urbanization in sub-Saharan Africa and implication for malaria control. Am J Trop Med Hyg. 2004;71(2 Suppl):118-127.

4. World Health Organization (WHO). World Malaria Report 2018. 11th ed. Geneva: WHO; 2018.

5. Mohamedahmed KA, Nour BY, Abakar AD, Babker AM. Diagnostic and prognostic value of thrombocytopenia severity in Sudanese children with Falciparum malaria. World J Adv Res Rev. 2020;6(3):197-204. doi:10.30574/ wjarr.2020.6.3.0196

6. World Health Organization (WHO). World Malaria Report 2016. Geneva: WHO; 2016.

7. Satoskar AR, Simon G, Hotez PJ, Tsuji M. Medical Parasitology. USA: Landes Bioscience; 2009:240-241.

8. Mohamedahmed KA, Ahmed ZA, Nour BY, Abakar AD, Babker AM. Impact of sever Plasmodium falciparum infection on platelets parameters among Sudanese children living in Al-Jazira state. Int J Clin Biomed Res. 2020;6(2):59. doi:10.31878/ijcbr.2020.62.02

9. Cheesbrough M. District Laboratory Practice in Tropical Countries, Part 1. 2nd ed. London: Cambridge University Press; 2006:240-242.

10. Phillips RS. Current status of malaria and potential for control. Clin Microbiol Rev. 2001;14(1):208-226. doi:10.1128/cmr.14.1.208-226.2001

11. World Health Organization (WHO). Basic Malaria Microscopy. 2nd ed. Geneva: WHO; 2010.

12. Kinra P, Dutta V. Serum TNF alpha levels: a prognostic marker for assessment of severity of malaria. Trop Biomed. 2013;30(4):645-653.

13. Black RE, Cousens S, Johnson HL, et al. Global, regional, and national causes of child mortality in 2008: a systematic analysis. Lancet. 2010;375(9730):1969-1987. doi:10.1016/ s0140-6736(10)60549-1

(C) 2020 The Author(s); This is an open-access article distributed under the terms of the Creative Commons Attribution License (http:// creativecommons.org/licenses/by/4.0), which permits unrestricted use, distribution, and reproduction in any medium, provided the original work is properly cited. 